\title{
Full-Length Infectious Clones of Two New Isolates of Tomato Mosaic Virus Induce Distinct Symptoms Associated with Two Differential Amino Acid Residues in 128-kDa Protein
}

\author{
Go-Woon Choi ${ }^{1 \dagger}$, June-Pyo Oh ${ }^{1 \dagger}$, In-Sook Cho ${ }^{2 \dagger}$, Hye-Kyoung Ju${ }^{1}$, Wen-Xing Hu ${ }^{1}$, Boram Kim ${ }^{1}$, Eun-Young \\ Seo $^{1}$, Jong-Seok Park ${ }^{3}$, Leslie L Domier ${ }^{4}$, John Hammond (ib ${ }^{5 *}$, Kihak Song ${ }^{6 *}$, and Hyoun-Sub Lim ${ }^{1 *}$ \\ ${ }^{1}$ Department of Applied Biology, College of Agriculture and Life Sciences, Chungnam National University, Daejeon \\ 34134, Korea \\ ${ }^{2}$ Horticultural and Herbal Crop Environment Division, National Institute of Horticultural and Herbal Science, RDA, \\ Wanju 55365, Korea \\ ${ }^{3}$ Department of Horticulture, College of Agriculture and Life Sciences, Chungnam National University, Daejeon 34134, \\ Korea \\ ${ }^{4}$ Department of Crop Sciences, University of Illinois at Urbana-Champaign, Urbana, IL 61801, USA \\ ${ }^{5}$ United States Department of Agriculture-Agricultural Research Service, Floral and Nursery Plants Research Unit, \\ Beltsville, MD 20705, USA \\ ${ }^{6}$ Department of Urology, Chungnam National University School of Medicine, Daejeon 34134, Korea
}

(Received on December 10, 2018; Revised on June 2, 2019; Accepted on June 10, 2019)

In 2017, two new tomato mosaic virus (ToMV) isolates were collected from greenhouses in Buyeo, Chungcheongnam-do, South Korea. Full-length cDNAs of the new ToMV isolates were cloned into dual cauliflower mosaic virus $35 \mathrm{~S}$ and $\mathrm{T} 7$ promoter-driven vectors, sequenced and their pathogenicities investigated. The nucleotide sequences of isolates GW1 (MH507165) and GW2 (MH507166) were 99\% identical, resulting in

\footnotetext{
${ }^{\dagger}$ These authors contributed equally to this work.

*Co-corresponding authors.

John Hammond

Phone) +1-301-504-5313, FAX) +1-301-504-5096

E-mail) john.hammond@ars.usda.gov

ORCID

https://orcid.org/0000-0001-7593-747X

Kihak Song

Phone) +82-42-280-7777, FAX) +82-42-823-8679

E-mail)songkihak62@gmail.com

Hyoun-Sub Lim

Tel) +82-42-821-5766, FAX) +82-42-823-8679

E-mail) hyounlim@cnu.ac.kr

(c) This is an Open Access article distributed under the terms of the Creative Commons Attribution Non-Commercial License (http:// creativecommons.org/licenses/by-nc/4.0) which permits unrestricted noncommercial use, distribution, and reproduction in any medium, provided the original work is properly cited.
}

Articles can be freely viewed online at www.ppjonline.org. only two amino acid differences in nonconserved region II and the helicase domain, Ile668Thr and Val834Ile. The two isolates were most closely related to a ToMV isolate from Taiwan (KJ207374). Isolate GW1 (Ile668, Val834) induced a systemic hypersensitive response in Nicotiana benthamiana compared with the isolate GW2, which a single residue substitution showed was due to Val834.

Keywords : difference of infectivity, full-length infectious cDNA clone, tobamovirus, tomato mosaic virus (ToMV)

Handling Editor : Yoon, Ju-Yeon

Production of tomato in Korea has increased in recent years, largely due to demands for phytonutrient-rich foods that are beneficial for human health and associated with reduced risks of diseases like diabetes and prostate cancer. Most tomatoes in Korea are greenhouse grown, where insect pests and seedborne diseases pose major threats to crop yield and quality. Viral diseases of tomato have been reported to affect up to $26.5 \%$ of tomato production (Choi et al., 2010). In a recent survey of tomato viral diseases, we found that the main causes of yield loss were tomato yellow leaf curl virus (TYLCV) and tomato mosaic virus 
(ToMV) (Choi et al., 2018). While TYLCV is transmitted by whiteflies, ToMV is transmitted mechanically or by contaminated seeds (Procter and Fry, 1965). Effective quarantine measures are required to prevent the introduction of ToMV-infected seeds through the international seed trade, as most tomato seeds planted in Korea are imported; there is a close relationship between ToMV incidence in tomato crops and sources of contaminated seed. Therefore, sequence analysis of new ToMV isolates can be used to determine their probable origin.

ToMV belongs to the genus Tobamovirus, family Virgaviridae. The genome of c.6400 nt encodes four proteins: the replicase components: the $128-\mathrm{kDa}$ and its $182-\mathrm{kDa}$ readthrough product from open reading frame (ORF) 1 and fusion protein from ORFs 1 and 2, respectively, the $30 \mathrm{kDa}$ cell-to-cell movement protein (MP) from ORF3, and the 17 $\mathrm{kDa}$ coat protein $(\mathrm{CP})$ from ORF4 (Ishibashi and Ishikawa, 2014). The $128-\mathrm{kDa}$ and $180-\mathrm{kDa}$ proteins are translated from the genomic RNA, whereas MP and CP are translated from 3' co-terminal subgenomic RNAs. The 128-kDa protein includes methyltransferase and helicase (HEL) domains (Buck, 1999), and has a silencing suppressor function, which inhibits production of small interfering RNAs and microRNAs before they can bind to RNA-induced silencing complexes (RISCs), although pre-assembled RISCs are not affected (Csorba et al., 2007). The silencing suppressor in the $128-\mathrm{kDa}$ protein targets both polarities of small RNAs, and small RNA duplexes originating from the viral genome (Kubota et al., 2003; Kurihara et al., 2007).
In 2017 greenhouse grown tomato leaf samples with virus-like symptoms were collected (Choi et al., 2018) and seven ToMV-infected tissue samples from three locations were used to construct full-length infectious cDNA clones. Total RNAs were extracted from pooled leaf tissues using TriZol reagent (Life Technologies, Carlsbad, CA, USA), and stored at $-70^{\circ} \mathrm{C}$ prior to use as templates; full-length cDNA was synthesized using a ReverTra Ace- $\alpha$ kit (Toyobo, Osaka, Japan) and a set of primers designed based on consensus sequence of a recently reported ToMV isolate; the $5^{\prime}$ primer included the $\mathrm{T} 7$ promoter sequence immediately upstream of the ToMV 5' sequence (Table 1). Polymerase chain reaction (PCR) amplification of complete ToMV genomes was performed using high-fidelity KOD FX NEO polymerase (Toyobo), with amplification conditions of $2 \mathrm{~min}$ at $94^{\circ} \mathrm{C}$, followed by 8 cycles of $10 \mathrm{~s}$ at $98^{\circ} \mathrm{C}, 30 \mathrm{~s}$ at $58^{\circ} \mathrm{C}$, and $5 \mathrm{~min}$ at $72^{\circ} \mathrm{C}$, and then 27 cycles of $10 \mathrm{~s}$ at $98^{\circ} \mathrm{C}, 30 \mathrm{~s}$ at $63^{\circ} \mathrm{C}$, and $5 \mathrm{~min}$ at $72^{\circ} \mathrm{C}$. The resulting amplicon was analyzed by electrophoresis in a $0.8 \%$ agarose gel and visualized using a transilluminator; the full-length product was then ligated at $4^{\circ} \mathrm{C}$ for $12 \mathrm{~h}$ to the T-Blunt vector (All In PCR cloning kit, BioFact, Daejeon, Korea) prior to transformation of Escherichia coli. Thirteen full-length clones were obtained and used to synthesize in vitro transcripts as described (Han et al., 2017). Transcripts from eight clones produced symptoms when inoculated to Nicotiana benthamiana, and only symptom-inducing cDNA clones were sequenced at Macrogen, Inc. (Seoul, Korea) using the primers shown in Table 1. Analysis of the

Table 1. Primers used in this study

\begin{tabular}{llc}
\hline \multicolumn{1}{c}{ Primer } & \multicolumn{1}{c}{ Sequence } & $\begin{array}{c}\text { Length } \\
\text { (bp) }\end{array}$ \\
\hline ToMV T7 BglII F & 5'-AAAAGATCTTAATACGACTCACTATAGGGGTATTTTTACAACAA TACAACAACAAC-3' & 58 \\
ToMV XmaI R & 5'-AAACCCGGGTGGGCCCCAACCGGGGGTTCC-3' & 30 \\
ToMV seq F 1 & 5'-CAGTATTGAACTATACCTTTCTA-3' & 23 \\
ToMV seq F 2 & 5'-GAAAGAATCTTGTTAGAGGA-3' & 20 \\
ToMV seq F 3 & 5'-GTCTCTCGAGCAGTTTCATAT-3' & 21 \\
ToMV seq F 4 & 5'-GACACGTGATGTGCACGTC-3' & 19 \\
ToMV seq R 1 & 5'-AAGGTTTCCACACCTCGCTG-3' & 20 \\
ToMV seq R 2 & 5'-CAGCAGCAGTGTAATATGAC-3' & 20 \\
ToMV seq R 3 & 5'-GCCAAGAAATCCTCTAGACC-3' & 20 \\
ToMV seq R 4 & 5'-CTAATAAGTAACTACTAACCCG-3' & 22 \\
ToMV overlap F & 5'-AAACTGCAGCAAAGGTAATAGTAGCAGTTATGAGCA-3' & 36 \\
ToMV overlap R & 5'-AAAGGATCCATCACAACGGTGTAGTACTT-3' & 29 \\
ToMV inner F T.V 5'-CTTCATACACTAGGAGCGA-3' & 19 \\
ToMV inner R T.V 5'-TCGCTCCTAGTGTATGAAG-3' & 19 \\
ToMV inner F V.I & 5'-GGTGGATGGCATTCCAG-3' & 17 \\
ToMV inner R V.I & 5'-CTGGAATGCCATCCACC-3' & 17 \\
\hline
\end{tabular}




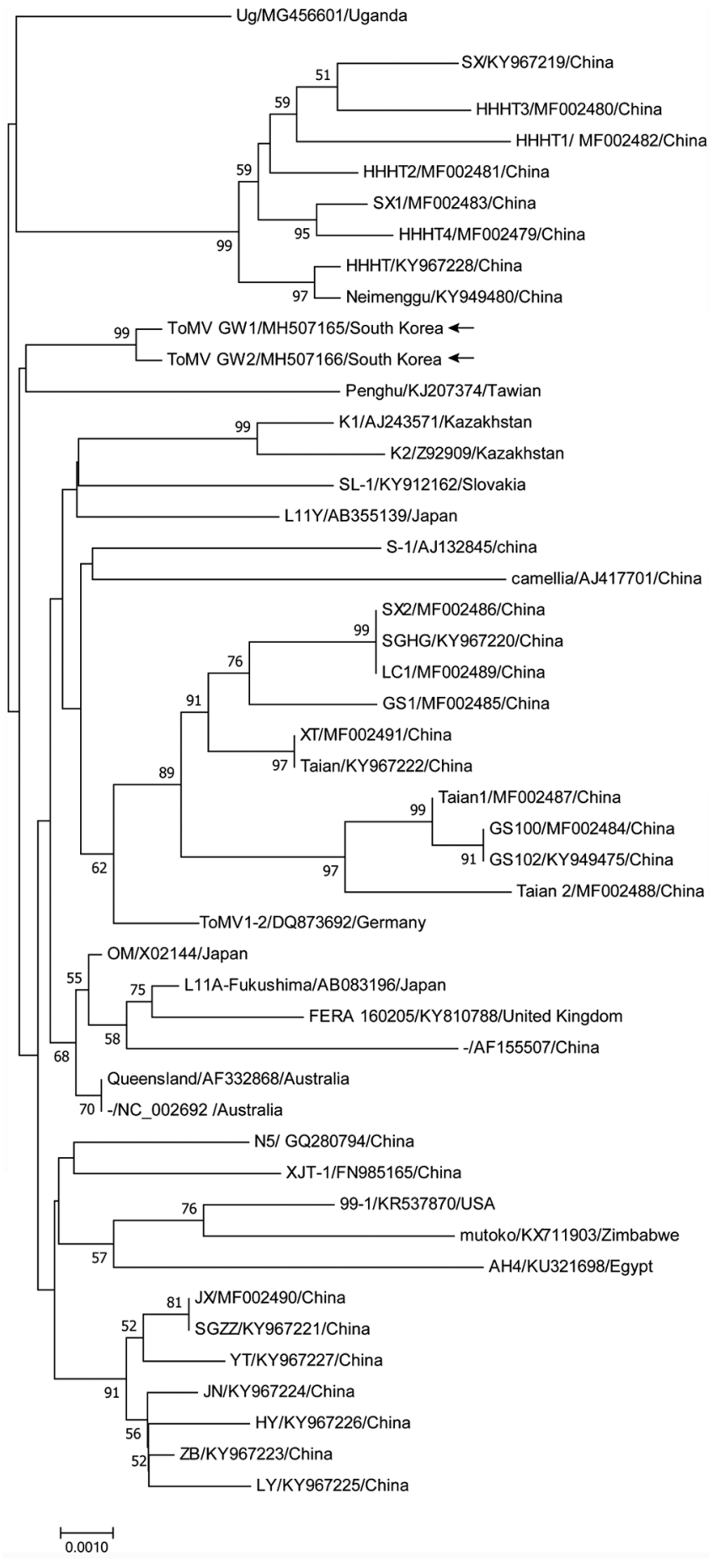

Fig. 1. Phylogenetic analysis of the new tomato mosaic virus (ToMV) isolates. The phylogenetic tree was constructed by the neighbor-joining method with 1,000 bootstrap replicates. The sequences of 45 ToMV isolates were selected from National Center for Biotechnology Information GenBank. The labels in the tree indicate name of isolates/accession number/country. The numbers at the nodes are bootstrap values above $50 \%$. The scale bar indicates the number of nucleotide substitutions. eight full-length clones showed that there were two distinct sequences, of which seven full-length cDNA clones were identified as GW1 (accession No. MH507165) and one as GW2 (accession No. MH507166). Isolates GW1 and GW2 both have genome sequences of 6,383 nt and share 99.95\% nt identity. Analysis using DNAMAN (Lynnon BioSoft, Quebec, Canada) showed that each contains the expected four ORFs that are predicted to encode the 128$\mathrm{kDa}, 182-\mathrm{kDa}$ readthrough protein, 30-kDa MP, and 17.5$\mathrm{kDa} \mathrm{CP}$. Only two amino acid residues differentiate the isolates, I668T and V834I in the $128-\mathrm{kDa}$ protein. The nucleotide sequences of isolates GW1 and GW2 and 45 ToMV sequences obtained from GenBank were analyzed phylogenetically using the neighbor-joining method in MEGA6 with 1,000 bootstrap replicates (Fig. 1). Isolates GW1 and GW2 were most closely related to Taiwanese isolate Penghu (KJ207374), with $99.22 \%$ and $99.23 \%$ identity, respectively. Similar comparisons of the predicted amino acid sequences from isolates GW1 and GW2 ORFs with those of ToMV-Penghu showed identities of $99.37 \%$ and $99.40 \%(128 \mathrm{kDa}) ; 99.28 \%$ and $99.30 \%(182 \mathrm{kDa})$; $99.62 \%$ for the MP of both, and $99.17 \%$ for the CP of both isolates.

To test the basis for the differences in pathogenicity of GW1 and GW2 and make it easier to inoculate plants with the cloned viruses, the full-length cDNAs were subcloned into the pJY vector (Park et al., 2017). First, the 5' portions, including the $\mathrm{T} 7$ promoter, were subcloned following digestion with BglII and PstI. The 3' portion of each clone was separately inserted following digestion with Pst $\mathrm{I}$ and $X m a \mathrm{I}$, to reassemble complete genomes of each isolate (Fig. 2A). Potential differences in infectivity and pathogenicity between isolates GW1 and GW2 resulting from the two variant amino acids at I668T and V834I in the $128-\mathrm{kDa}$ protein were examined by transforming the respective $\mathrm{pJY}$ constructs into Agrobacterium tumefaciens strain GV2260, and agroinfiltrating leaves of 20-day-old $N$. benthamiana plants as described (Han et al., 2017). In addition, in vitro transcripts generated by the method of Kim et al. (2017) were inoculated to Solanum lycopersicum. Plants were grown with a $14 \mathrm{~h}$ photoperiod at $26^{\circ} \mathrm{C}$. At 7 days post inoculation (dpi), GW1 induced apical necrosis with a systemic hypersensitive response in $N$. benthamiana, whereas GW2 produced mild mosaic symptoms at 20 dpi (Fig. 2B). In S. lycopersicum, GW1 and GW2 both produced very mild symptoms (data not shown). Hence, the two amino acid differences between isolates GW1 and GW2 were associated with different symptoms in $N$. benthamiana, but not in S. lycopersicum. Overlap extension PCR (Higuchi et al., 1988) was used to separately substitute amino acid 
A
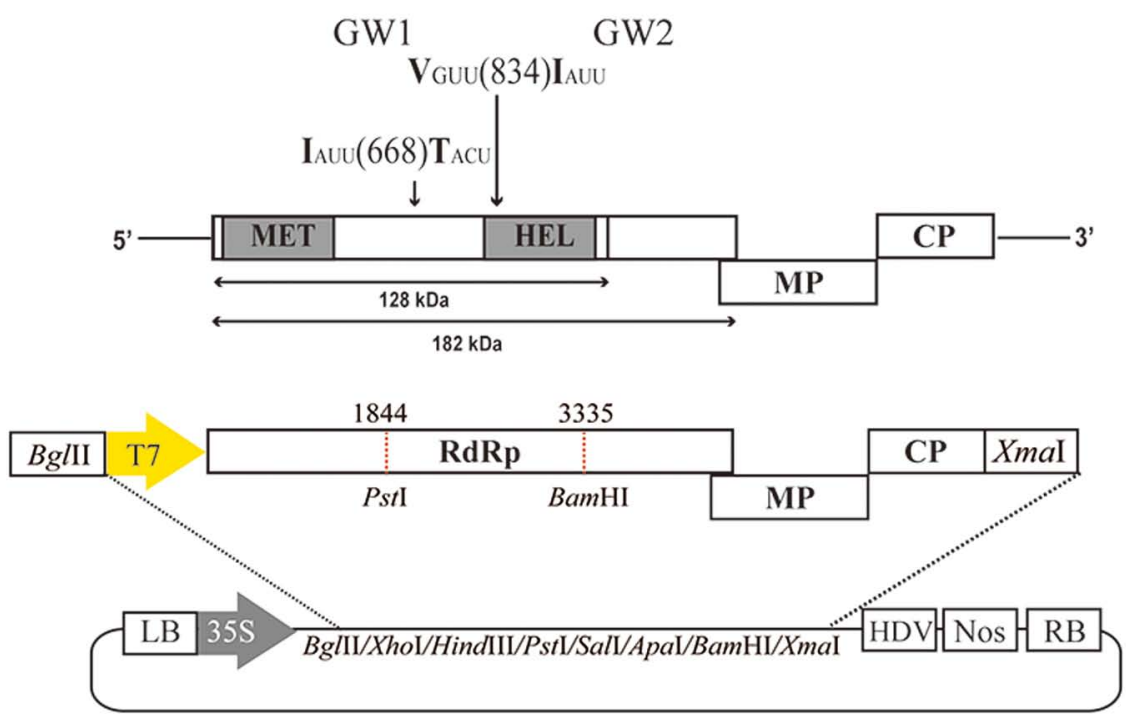

Topo vector

(S. lycopersicum)

pJY vector (N. benthamiana)

B

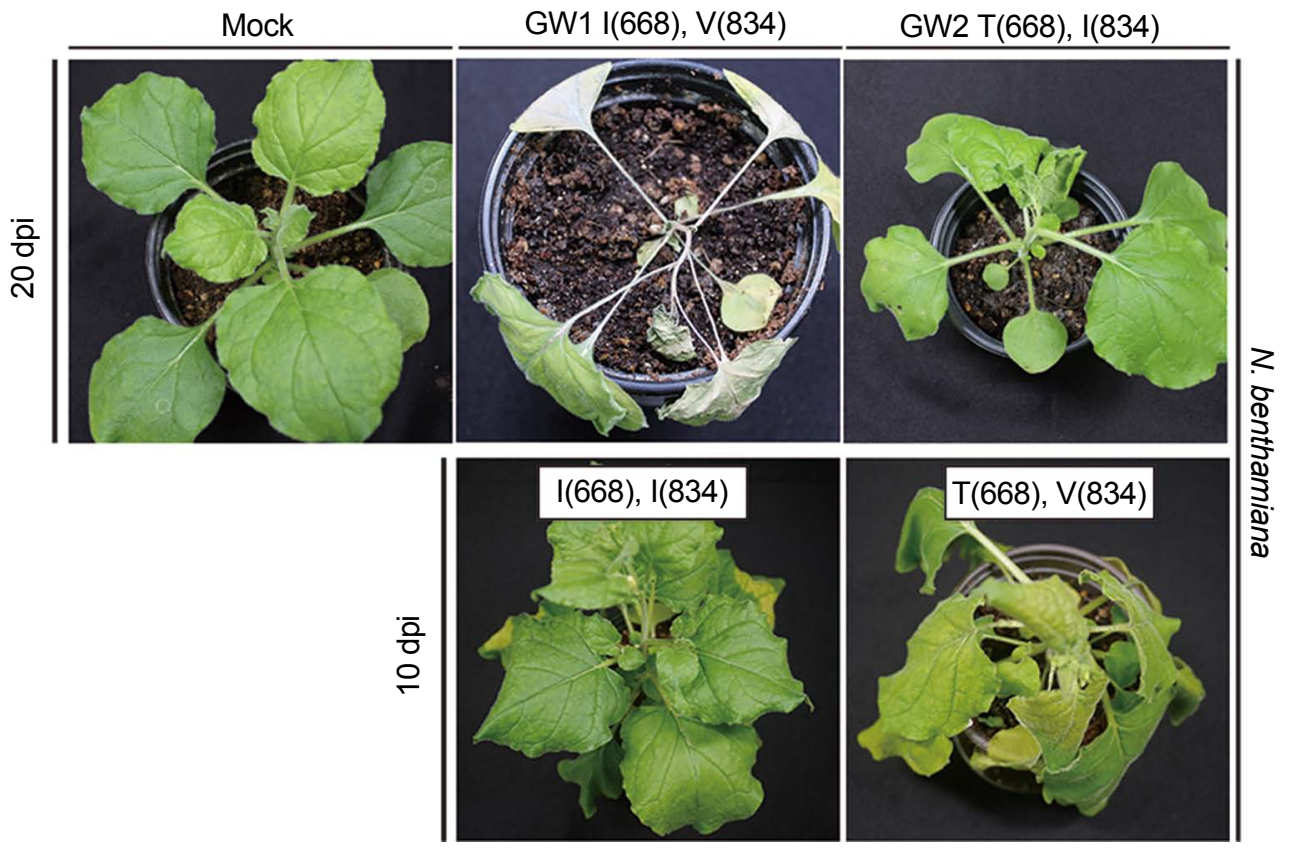

Fig. 2. Construction of tomato mosaic virus (ToMV) infectious clones in T-Blunt vector and binary vector, and symptoms induced by ToMV GW1 and GW2. (A) Total RNA was extracted from seven pooled ToMV-infected samples, and cDNA synthesized using ToMV-specific $3^{\prime}$ end and random primer. To generate infectious cDNA clones, full-length cDNA was amplified by polymerase chain reaction (PCR) using ToMV-specific primer sets (Table 1). The upstream primer included a T7 promoter and BglII enzyme site, and the downstream primer added an XmaI enzyme site. The full-length ToMV PCR product was cloned into the T-blunt vector. The full-length nucleotide sequences, and the predicted amino acid sequences of ToMV GW1 and GW2 open reading frames were compared. The only two amino acid differences between the two isolates occurred in the $128 \mathrm{kDa}$ protein. Full-length ToMV cloned T-Blunt vector was digested with BglII and PstI restriction enzymes. First, the $5^{\prime}$ fragment $(1.8 \mathrm{~kb}$ ) was inserted in pJY vector (Han et al., 2017). Subsequently, using PstI and XmaI restriction enzymes, the 4.6-kb $3^{\prime}$ fragment was cloned in $\mathrm{pJY}$ vector already containing the $5^{\prime}$ fragment. The two infectious clones were designated ToMV GW1 and GW2 and were inoculated to Nicotiana benthamiana either by using in vitro transcripts or agroinfiltration. The nucleotide sequence differences resulting in amino acid differences at residues 668 and 834 are indicated. (B) The infectious clones of isolates GW1 and GW2, and of mutants GW1-V834I and GW1-I668T of ToMV were each inoculated to $N$. benthamiana by agroinfiltration at the in same concentration (O.D. $\left.{ }_{600}\right)$, to plants at the 3-4 leaf stage. Symptoms were recorded at the indicated days post inoculation (dpi). Virus infections were confirmed by reverse transcriptase polymerase chain reaction. 
residues 668 and 834 of GW1 to distinguish the effects of each. Mutagenic primers were designed to change Ile 668 to Thr (GW1-I668T) and Val 834 to Ile (GW1-V834I) respectively (Table 1), with plasmid pJY:ToMV-GW1 as the template for each substitution. PCR mixtures contained $4 \mu \mathrm{l}$ template, $1.5 \mu \mathrm{l}$ of $10 \mathrm{pmol}$ each primer, $10 \mu \mathrm{l} 2 \mathrm{mM}$ dNTPs, $25 \mu 12 \times$ PCR buffer for KOD FX Neo, $1 \mu \mathrm{l}$ of KOD FX Neo and $7 \mu 1$ distilled water. PCR conditions were as follows: $2 \mathrm{~min}$ at $94^{\circ} \mathrm{C}, 30$ cycles of $10 \mathrm{~s}$ at $98^{\circ} \mathrm{C}$, $30 \mathrm{~s}$ at $58^{\circ} \mathrm{C}$, and $30 \mathrm{~s}$ at $68^{\circ} \mathrm{C}$. Products were gel-extracted and purified; $2 \mu 1$ of $5^{\prime}$ and $3^{\prime}$ GW1 mutant PCR products in equal molar ratio were used as PCR template with $1.5 \mu \mathrm{l}$ of 10 pmol ToMV overlap F/ToMV overlap $R$ as described above. Amplified GW1 mutants were separately ligated into gel-extracted pJY:ToMV-GW1 digested with PstI and BamHI (located at nt1844 and nt3335). The symptoms induced by pJY:ToMV-GW1 mutants were compared to the wild-type ToMV-GW1 and ToMV-GW2; the mutants were transformed into A. tumefaciens and agroinfiltrated to $N$. benthamiana. Symptoms were observed from 7 days post agroinfiltration. Mutant GW1-V834I (in the HEL domain) yielded mild symptoms similar to those induced by GW2, whereas mutant GW1-I668T induced severe symptoms, including systemic necrosis at $10 \mathrm{dpi}$, clearly linking HEL residue V834 to the induction of systemic necrosis (Fig. 2B). Multiple studies have shown that the tobamoviral 128$\mathrm{kDa}$ protein plays a role in symptom severity because of changes in viral replication and RNA silencing suppressor function. Especially, variations in symptom development were mapped to amino acid substitutions in nonconserved region II (NONII, equivalent to residues 454-806 of TMV) (Bao et al., 1996) and the HEL domain of the 128-kDa protein (Kubota et al., 2003; Wang et al., 2012). Our finding that two isolates with amino acid differences in HEL and NONII show different symptoms in $N$. benthamiana are consistent with prior results of other groups and clearly link HEL residue V834 to the induction of systemic necrosis, reinforcing the importance of the multifunctional $128-\mathrm{kDa}$ protein function in the Tobamovirus replication cycle (Bao et al., 1996; Kubota et al., 2003; Wang et al., 2012).

\section{Acknowledgments}

This work was supported by a grant from the Next-Generation BioGreen 21 Program (Project No. PJ01365501), Rural Development Administration, Republic of Korea.

\section{References}

Bao, Y., Carter, S. A. and Nelson, R. S. 1996. The 126- and 183-kilodalton proteins of tobacco mosaic virus, and not their common nucleotide sequence, control mosaic symptom formation in tobacco. J. Virol. 70:6378-6383.

Buck, K. W. 1999. Replication of Tobacco mosaic virus RNA. Philos. Trans. R. Soc. Lond. B Biol. Sci. 354:613-627.

Choi, G.-W., Kim, B., Ju, H., Cho, S., Seo, E., Kim, J., Park, J., Hammond, J. and Lim, H.-S. 2018. Dual infections of Tomato mosaic virus (ToMV) and Tomato yellow leaf curl virus (TYLCV), or Tomato mosaic virus (ToMV) and Tomato chlorosis virus (ToCV), detected in tomato fields located in Chungcheongnam-do in 2017. Korean J. Agric. Sci. 45:38-42.

Choi, H. S., Lee, S. H., Kim, M. K., Kwak, H. R., Kim, J. S., Cho, J. D. and Choi, G. S. 2010. Occurrence of virus diseases on major crops in 2009. Res. Plant Dis. 16:1-9 (in Korean).

Csorba, T., Bovi, A., Dalmay, T. and Burgyán, J. 2007. The p122 subunit of Tobacco mosaic virus replicase is a potent silencing suppressor and compromises both small interfering RNAand microRNA-mediated pathways. J. Virol. 81:1176811780.

Han, S.-H., Park, J.-S., Han, J.-Y., Gong, J.-S., Park, C.-H., Kim, J.-K., Seo, E.-Y., Domier, L. L., Hammond, J. and Lim, H.-S. 2017. New Korean isolates of Pepper mild mottle virus (PM$\mathrm{MoV})$ differ in symptom severity and subcellular localization of the $126 \mathrm{kDa}$ protein. Virus Genes 53:434-445.

Higuchi, R., Krummel, B. and Saiki, R.K. 1988. A general method of in vitro preparation and specific mutagenesis of DNA fragments: study of protein and DNA interactions. Nucleic Acids Res. 16:7351-7367.

Ishibashi, K. and Ishikawa, M. 2014. Mechanisms of tomato mosaic virus RNA replication and its inhibition by the host resistance factor Tm-1. Curr. Opin. Virol. 9:8-13.

Kim, I.-H., Han, J.-Y., Cho, I.-S., Ju, H. K., Moon, J. S., Seo, E.-Y., Kim, H. G., Hammond, J. and Lim, H.-S. 2017. Generation of an infectious clone of a new Korean isolate of Apple chlorotic leaf spot virus driven by dual $35 \mathrm{~S}$ and $\mathrm{T} 7$ promoters in a versatile binary vector. Plant Pathol. J. 33:608-613.

Kubota, K., Tsuda, S., Tamai, A. and Meshi, T. 2003. Tomato mosaic virus replication protein suppresses virus-targeted posttranscriptional gene silencing. J. Virol. 77:11016-11026.

Kurihara, Y., Inaba, N., Kutsuna, N., Takeda, A., Tagami, Y. and Watanabe, Y. 2007. Binding of tobamovirus replication protein with small RNA duplexes. J. Gen. Virol. 88(Pt. 8):2347-2352.

Park, C.-H., Ju, H.-K., Han, J.-Y., Park, J.-S., Kim, I.-H., Seo, E.Y., Kim, J.-K., Hammond, J. and Lim, H.-S. 2017. Complete nucleotide sequences and construction of full-length infectious cDNA clones of cucumber green mottle mosaic virus (CGMMV) in a versatile newly developed binary vector including both 35S and T7 promoters. Virus Genes 53:286-299.

Procter, C. H. and Fry, P. R. 1965. Seed transmission of tobacco mosaic virus in tomato. N. Z. J. Agric. Res. 8:367-369.

Wang, L.-Y., Lin, S.-S., Hung, T.-H., Li, T.-K., Lin, N.-C. and Shen, T.-L. 2012. Multiple domains of the Tobacco mosaic virus $\mathrm{p} 126$ protein can independently suppress local and systemic RNA silencing. Mol. Plant-Microbe Interact. 25:648-657. 